\title{
An Asymmetric 4-PAM Transceiver with 5.6-Gb/s/channel Data Rate for Display Interface with Ultra-high Definition
}

\author{
Kwang-Hun Lee and Young-Chan Jang \\ Kumoh National Institute of Technology, Gumi, Korea \\ ycjang@kumoh.ac.kr
}

\begin{abstract}
A 5.6-Gb/s/channel 4-pulse amplitude modulation (PAM) transceiver is designed for a high-bandwidth chip-to-chip interface in a display application. The asymmetric 4-PAM signaling scheme is proposed to increase the voltage and time margins, and the implemented scheme reduces the reference noise effect in a receiver by 33\%. The proposed asymmetric 4PAM transceiver is implemented by using a 0.13- $\mu$ m 1-poly 6-metal CMOS process with a 1.2 $V$ supply. The measured rms jitter of the output clock of the phase-locked loop (PLL) is 4.18 ps at the operating frequency of $700 \mathrm{MHz}$ for 5.6-Gb/s/channel with a quad data rate scheme. The measurement results show that the proposed asymmetric 4-PAM signaling increases the voltage margin by $23.5 \%$ without reduction in the time margin as compared with conventional 4-PAM signaling when the noise magnitude of the single reference is $65 \mathrm{mV}$. The active area and power consumption of a 1-channel transceiver including the PLL are $0.294 \mu^{2}$ and $6 \mathrm{~mW} / \mathrm{Gb} / \mathrm{s}$, respectively.
\end{abstract}

Keywords: Asymmetric, 4-PAM, transceiver, receiver, reference, display application

\section{Introduction}

As the resolution of display system is changed from a full-high definition (FHD) to an ultra-high definition (UHD), demands for a display interface with higher bandwidth have been increasing. For a high-bandwidth interface, the design techniques used in high-speed serial links become more important. For example, design techniques such as data training, transmitter (TX)/receiver (RX) equalization, incorporation of circuits with inductors, and data coding have been adopted or considered for a high-speed chip-to-chip interface system [1-3]. In this context, a pulse amplitude modulation (PAM) is another technique that can be implemented in a high-bandwidth display interface system [4]. In general, 4-PAM signaling increases the timing margin at the receiver as it reduces the Nyquist frequency for the interface channel by half. However, in terms of the voltage margin, 4-PAM signaling is effective when more than $10 \mathrm{~dB}$ of channel loss, mostly caused by dielectric loss and skin effect, is expected by doubling the signaling speed [4]. This is because 4-PAM signaling itself reduces the voltage margin by one-third.

Multi-level signaling is effective for the reduction of not only intrinsic channel loss but also power consumption. Figure 1 shows the simulated power consumption of the differential 2-PAM and 4-PAM transceivers in the point-to-point channel with a length of $30 \mathrm{~cm}$. As the data rate increases beyond $5-\mathrm{Gb} / \mathrm{s} / \mathrm{channel}$, the power efficiency of the 4-PAM transceiver increases with respect to that of the 2-PAM transceivers. This is because the 2-PAM transceivers (two types of 4:1 TXMUX and termination) need more power to compensate for the loss of SNR by heavy inter-symbol-interference (ISI) in their internal nodes. The usage of 
4-PAM signaling also reduces the power consumption of the clock tree because of its low operation frequency compared to the 2-PAM.

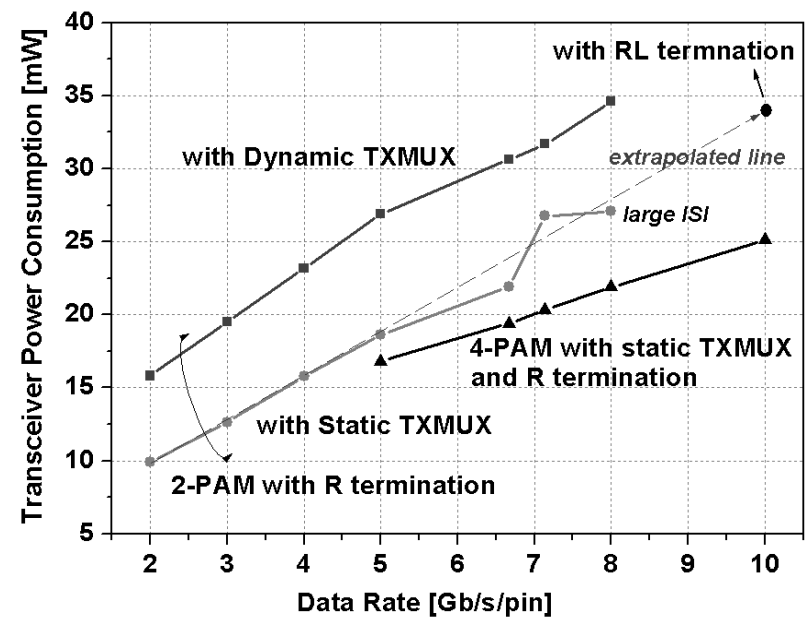

Figure 1. Power consumption of transceiver except clock tree

Figure 2 shows the block diagram for one channel of a conventional 4-PAM transceiver. The TX is composed of two drivers with different current sources, as shown in Figure 3(a). In general, a driver for the least significant bit (LSB) has a current source of half of that of the most significant bit (MSB). Further, each driver uses a differential amplifier for differential signaling. The RX is composed of three unit receivers for the 4-PAM, as shown in Figure 2. The RX, with the reference shown in Figure 3(b), is used for RX0_H and RX0_L, as shown in Figure 2. The RX shown in Figure 3(c) is used only for RX0_M to determine the MSB. In addition, a logic decoder block is required to convert the thermometer code to the binary code for the 4-PAM.

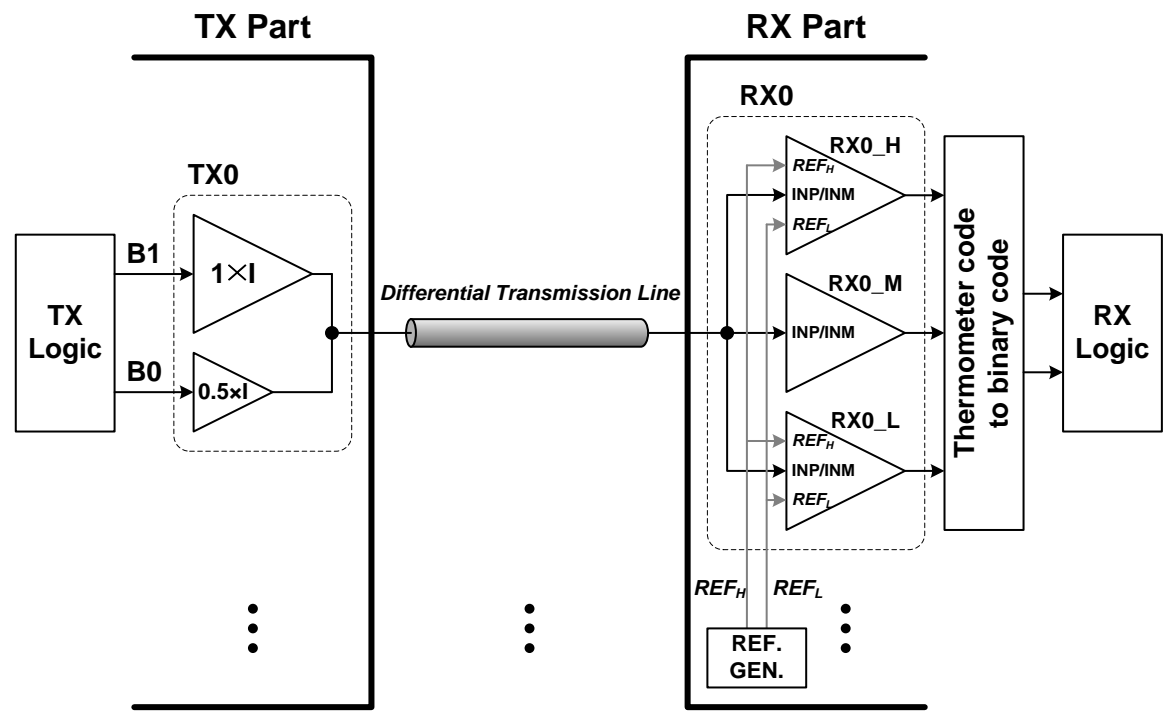

Figure 2. Block diagram of conventional 4-PAM transceiver 


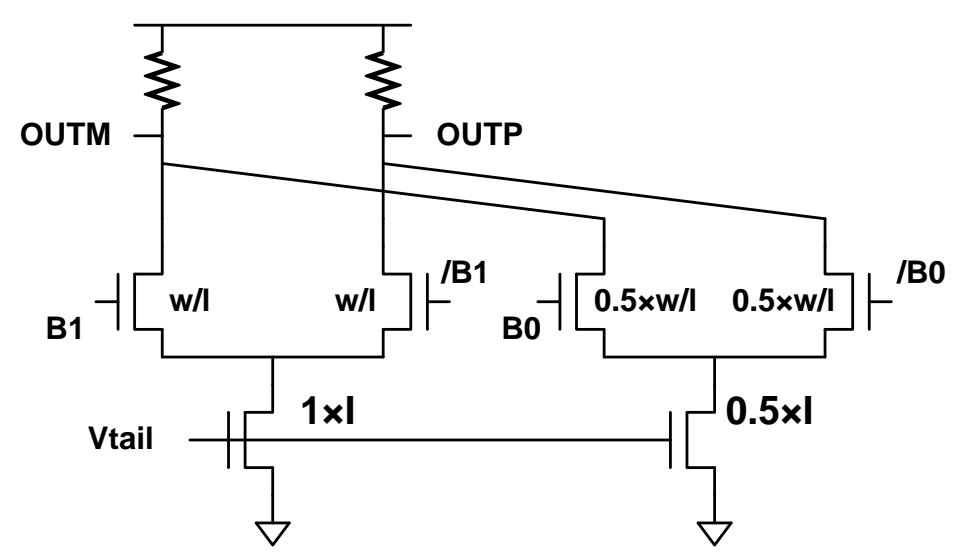

(a)

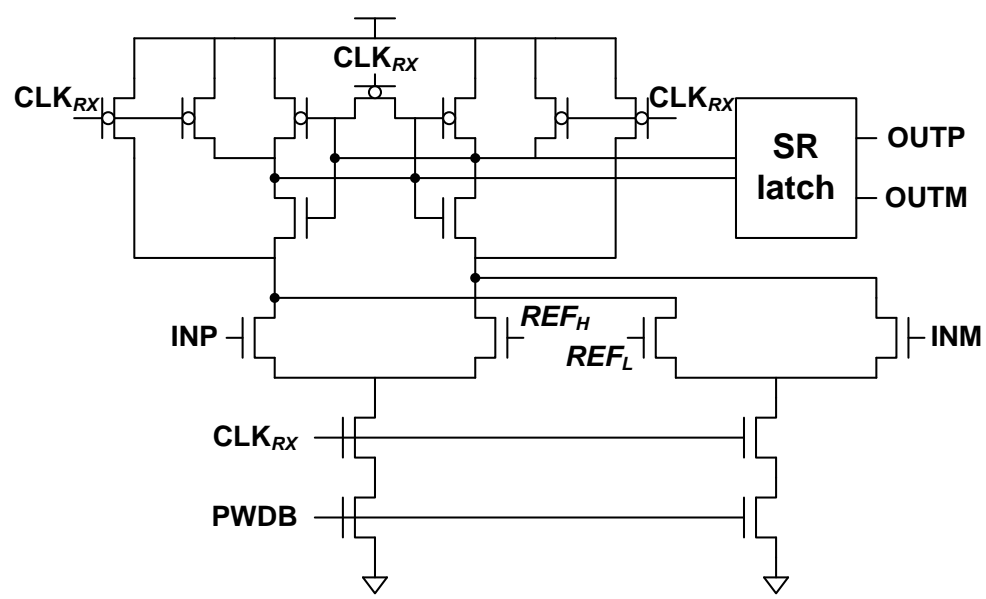

(b)

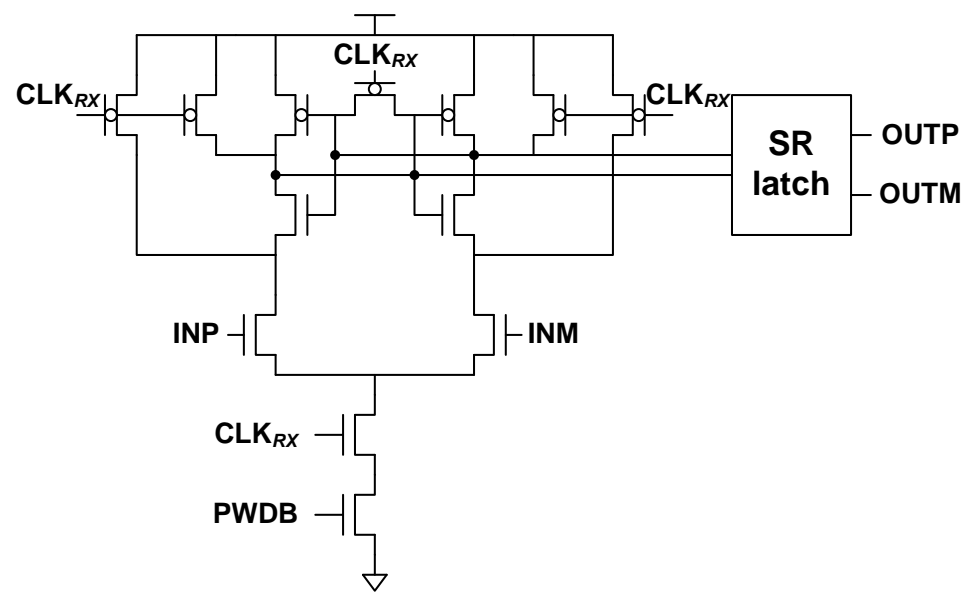

(c)

Figure 3. 4-PAM transceiver circuit (a) transmitter (b) receiver with reference $(c)$ receiver without reference

Figure 4 shows the conceptual eye diagram of a conventional 4-PAM [4]. In the conventional 4-PAM that has $\Delta \mathrm{V}_{2}, \Delta \mathrm{V}_{1}$, and $\Delta \mathrm{V}_{0}$ of the same voltage amplitude $(\Delta \mathrm{V})$, the 
reference noise decreases the voltage margin in RX0_H and RX0_L. Equations (1)-(4) show the voltage margin for the four cases in RX0_H. When the deterministic noise magnitude of the references $\left(\mathrm{REF}_{\mathrm{H}}, \mathrm{REF}_{\mathrm{L}}\right)$ is $3 \alpha$, the voltage margin for the worst case is $\Delta \mathrm{V}$ minus $6 \alpha$. The voltage margin in RX0_H is equal to that of RX0_L. However, in the case of RX0_M, the voltage margin for the worst case is $\triangle \mathrm{V}$ because $\mathrm{RX} 0 \_\mathrm{M}$ does not use any reference, as shown in Figure 3(c). Moreover, the time margin was determined in RX0_H and RX0_L because $T_{\text {eye2 }}$ is smaller than $T_{\text {eye1 }}$ due to the inherent feature of 4-PAM. As a result, the voltage and time margin in the 4-PAM receiver are determined by the signal integrity in the front of the RX and the performance of the RX with the input stage for the reference.

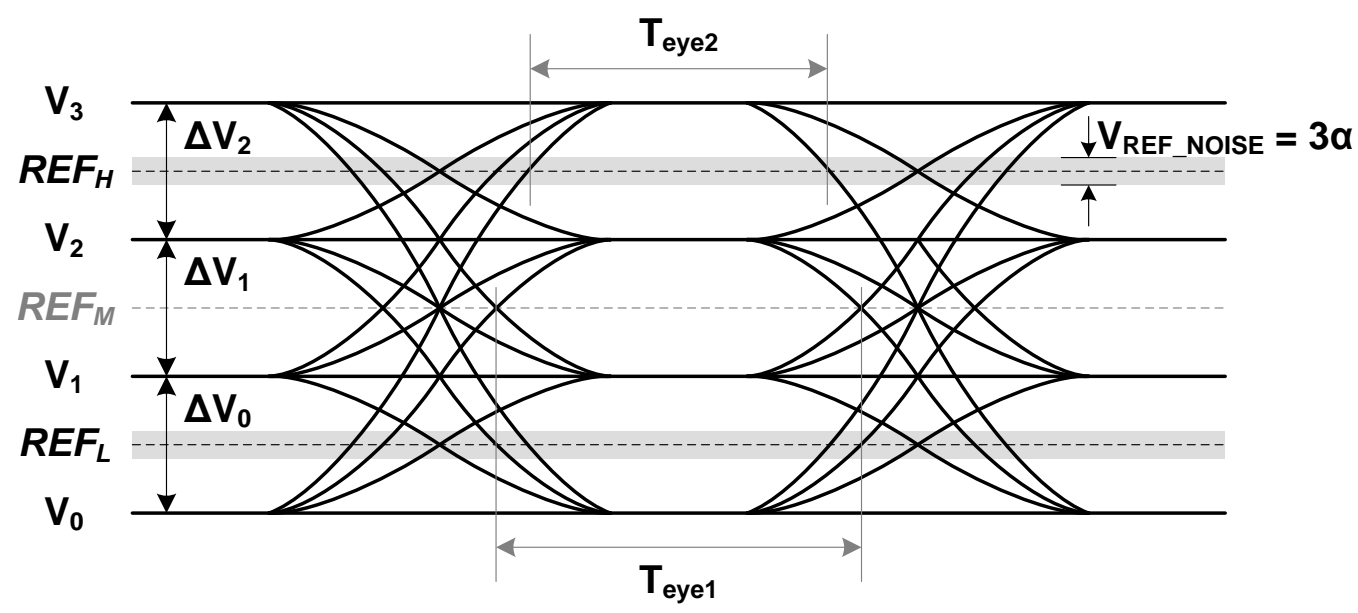

Figure 4. Conceptual eye diagram of conventional 4-PAM

$$
\begin{aligned}
V_{\text {MARGIN-CASE1-RX } 0_{-} H} & =\left|\left(V_{3}-R E F_{H}\right)-\left(V_{0}-R E F_{L}\right)\right|-2 \times 3 \alpha \\
& =|(0.5 \cdot \Delta V)-(-0.5 \cdot \Delta V)|-6 \alpha \\
& =\Delta V-6 \alpha \\
V_{\text {MARGIN-CASE } 2-R X_{0} 0_{-}} & =\left|\left(V_{2}-R E F_{H}\right)-\left(V_{1}-R E F_{L}\right)\right|-2 \times 3 \alpha \\
& =|(-0.5 \cdot \Delta V)-(0.5 \cdot \Delta V)|-6 \alpha \\
& =\Delta V-6 \alpha \\
& =\left|\left(V_{1}-R E F_{H}\right)-\left(V_{2}-R E F_{L}\right)\right|-2 \times 3 \alpha \\
& =|(-1.5 \cdot \Delta V)-(1.5 \cdot \Delta V)|-6 \alpha \\
& =3 \cdot \Delta V-6 \alpha \\
V_{\text {MARGIN-CASE3-RX } 0_{-} H} & =\left|\left(V_{0}-R E F_{H}\right)-\left(V_{3}-R E F_{L}\right)\right|-2 \times 3 \alpha \\
V_{\text {MARGIN-CASE4-RX } 0_{-} H} & =|(-2.5 \cdot \Delta V)-(2.5 \cdot \Delta V)|-6 \alpha \\
& =5 \cdot \Delta V-6 \alpha
\end{aligned}
$$

In this work, the asymmetric 4-PAM signaling scheme, which allows better voltage and timing margins at a $5.6-\mathrm{Gb} / \mathrm{s} / \mathrm{channel}$ data rate by controlling the eye opening of each signal 
level asymmetrically, is proposed. This paper is organized as follows. Section 2 describes the concept of the proposed 4-PAM transceiver and circuit implementation along the simulation results. Section 3 examines its chip implementation and measurement results. Section 4 concludes the paper.

\section{Asymmetric 4-PAM Transceiver}

\subsection{Concept of proposed Asymmetric 4-PAM}

In order to reduce the reference noise effect generated in the receiver of the conventional 4-PAM, the asymmetric 4-PAM, whose conceptual eye diagram is shown in Figure 5, is proposed in this work. When the deterministic noise magnitude of the references is assumed to be $3 \alpha$, the levels of $V_{1}$ and $V_{2}$ are adjusted by $2 \alpha$ and the level of reference is adjusted by $1 \alpha$. In this case, the power consumption of the transceiver is equal to that of the conventional 4-PAM, because the amplitude of the maximum full swing from $V_{0}$ to $V_{3}$ does not change.

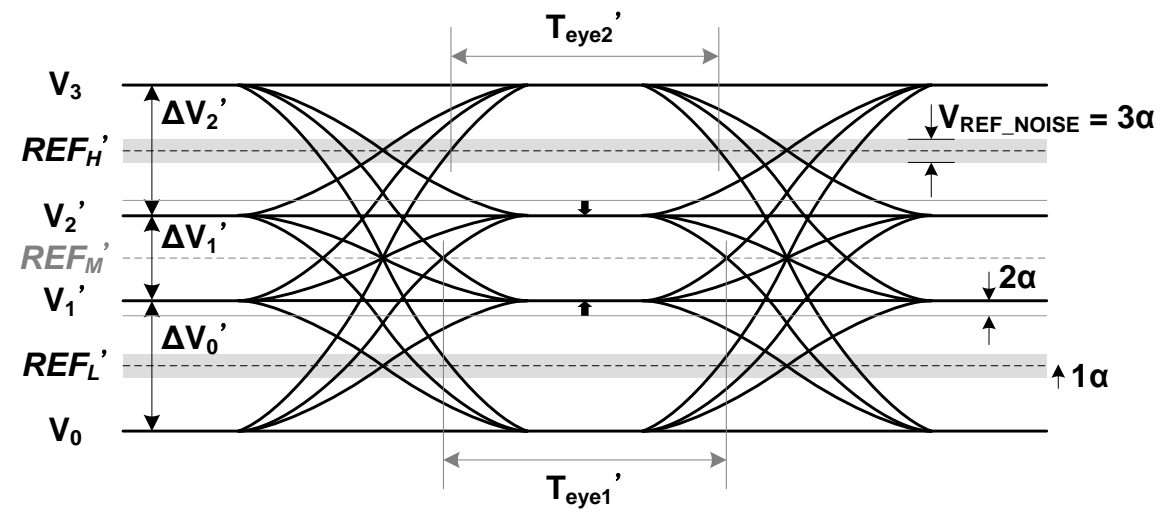

Figure 5. Conceptual eye diagram of asymmetric 4-PAM

$$
\Delta V-\beta=\left(\Delta V+\frac{\beta}{N-2}\right)-2 \times \delta \quad \beta=\frac{2 \delta \cdot(N-2)}{N-1}
$$

The optimal levels of $\mathrm{V}_{1}^{\prime}$ and $\mathrm{V}_{2}^{\prime}$ for the asymmetric 4-PAM are determined by the condition that the magnitude of the center eye is equal to that of the upper or lower eye when including the effect of reference noise. Equation (5) is the generalized formula for the $N$-PAM, where $\beta$ denotes the summation of magnitude at which each level of $\mathrm{V}_{1}$ and $\mathrm{V}_{2}$ is controlled. The term $N$ denotes the multi-level number of PAM and $\delta$ denotes the noise magnitude of the reference signal. When $\delta$ is equal to $3 \alpha$ in $4-\mathrm{PAM}, \beta$ is determined to be $4 \alpha$. Equations (6)(13) show the voltage margin for four cases of RX0_H and RX0_M in the asymmetric 4PAM with the reference noise of $3 \alpha$. The voltage margin for the worst case is $\Delta \mathrm{V}$ minus $4 \alpha$. The voltage margin of $2 \alpha$ was increased compared to the conventional 4-PAM. Further, the time margin in RX0_H increased from $\mathrm{T}_{\text {eye } 2}$ to $\mathrm{T}_{\text {eye } 2}{ }^{\prime}$ by shifting the reference level $\left(\mathrm{REF}_{\mathrm{H}}{ }^{\prime}\right.$, $\mathrm{REF}_{\mathrm{L}}^{\prime}$ ).

$$
\begin{aligned}
V_{\text {MARGIN-CASEI-RX } 0_{-} H} & =\left|\left(V_{3}-R E F_{H}{ }^{\prime}\right)-\left(V_{0}-R E F_{L}{ }^{\prime}\right)\right|-2 \times 3 \alpha \\
& =|(0.5 \cdot \Delta V+1 \alpha)-(-0.5 \cdot \Delta V-1 \alpha)|-6 \alpha \\
& =\Delta V-4 \alpha
\end{aligned}
$$




$$
\begin{aligned}
& V_{\text {MARGIN-CASE2-RX } 0_{-} H}=\left|\left(V_{2}^{\prime}-R E F_{H}{ }^{\prime}\right)-\left(V_{1}^{\prime}-R E F_{L}{ }^{\prime}\right)\right|-2 \times 3 \alpha \\
& =|(-0.5 \cdot \Delta V-1 \alpha)-(0.5 \cdot \Delta V+1 \alpha)|-6 \alpha \\
& \left.V_{\text {MARGIN-CASE3-RX } 0_{-} H}=\mid \Delta V_{1}^{\prime}-4 \alpha F_{H}{ }^{\prime}\right)-\left(V_{2}{ }^{\prime}-R E F_{L}{ }^{\prime}\right) \mid-2 \times 3 \alpha \\
& =|(-1.5 \cdot \Delta V+3 \alpha)-(1.5 \cdot \Delta V-3 \alpha)|-6 \alpha \\
& =3 \cdot(\Delta V-4 \alpha) \\
& V_{\text {MARGIN-CASE4-RX } 0_{-} H}=\left|\left(V_{0}-R E F_{H}{ }^{\prime}\right)-\left(V_{3}-R E F_{L}^{\prime}\right)\right|-2 \times 3 \alpha \\
& =|(-2.5 \cdot \Delta V+1 \alpha)-(2.5 \cdot \Delta V-1 \alpha)|-6 \alpha \\
& =5 \cdot \Delta V-8 \alpha \\
& V_{M A R G I N-C A S E 1_{-R X} 0_{-} M}=\left|V_{3}-V_{0}\right|=3 \cdot \Delta V \\
& V_{\text {MARGIN-CASE2-RX } 0_{-} M}=\left|V_{2}{ }^{\prime}-V_{1}{ }^{\prime}\right|=1 \cdot \Delta V-4 \alpha \\
& V_{\text {MARGIN-CASE3-RX } 0_{-} M}=\left|V_{1}{ }^{\prime}-V_{2}{ }^{\prime}\right|=1 \cdot \Delta V-4 \alpha \\
& V_{\text {MARGIN-CASE4-RX } 0_{-} M}=\left|V_{0}-V_{3}\right|=3 \cdot \Delta V
\end{aligned}
$$

For the proposed asymmetric 4-PAM scheme, the transmitter adjusts the current strength of the TX driver for the LSB (B0), as shown in Figure 6. In this work, the transmitter is constituted of identical cells described in the following section, and it can adjust the current strength of both the MSB and LSB drivers.

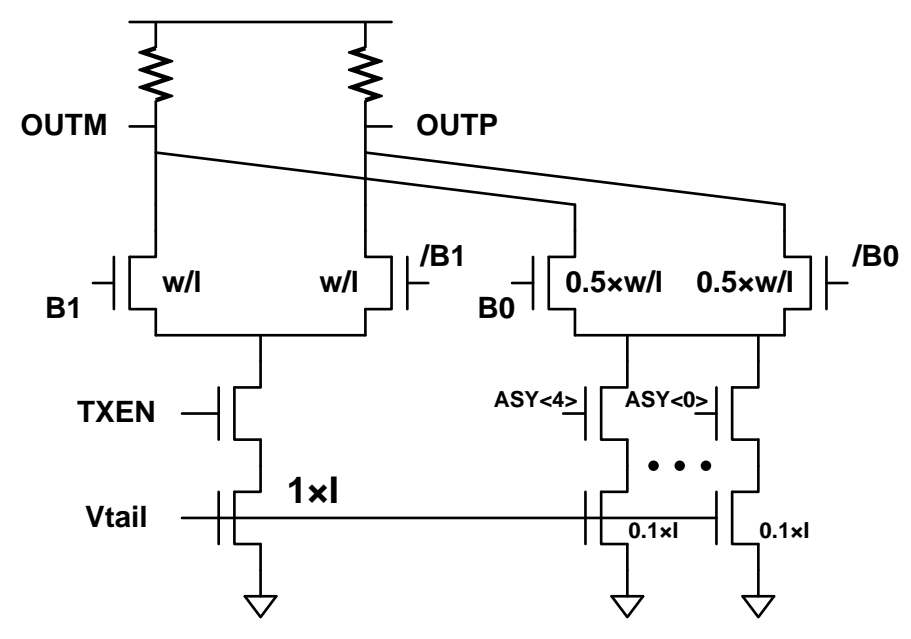

Figure 6. Transmitter for asymmetric 4-PAM

\subsection{Circuit Implementation}

Figure 7 shows the block diagram of the implemented transceiver for the proposed asymmetric 4-PAM with a quad data rate (QDR). The TX part consists of the test block that generates 4-bit data from an external pseudo random bit sequence generator, a phase-locked loop (PLL), and a total of 20 TX cells along with 1-tap pre-emphasis that is $20 \mathrm{~dB}$ of MSB and LSB drivers to reduce channel ISI [5]. MSB and LSB drivers are segmented to 20 identical TX cells to reduce power consumption according to signal integrity and 
asymmetrically control the eye diagram size. Twelve TX cells are used for the MSB driver and six TX cells are used for the LSB driver. The remaining cells are used for pre-emphasis of each driver. The number of TX cells for MSB and LSB in turn on depends on signal integrity.

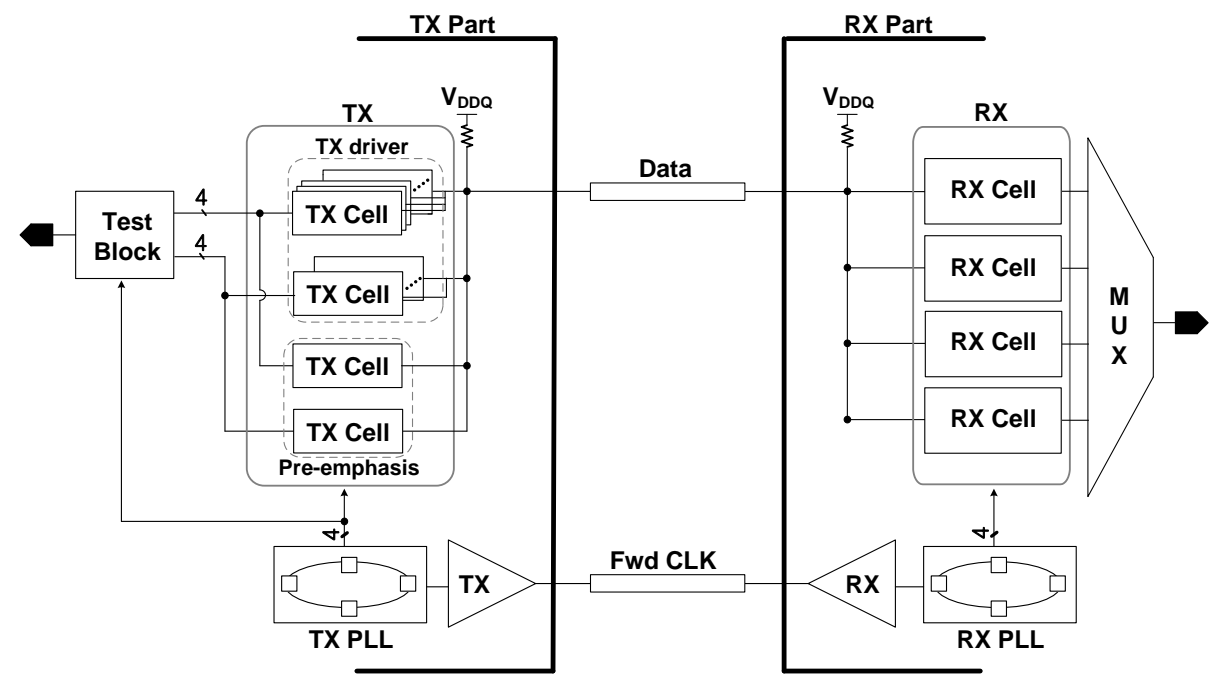

Figure 7. The block diagram of the implanted asymmetric 4-PAM transceiver

Figure 8 shows the schematic of the TX cell. It consists of four-pulse generators, a predriver, and a main driver. The four-pulse generates 4-bit data that is generated by the test block when $\mathrm{CLK}_{\mathrm{i}}$ and $\mathrm{CLK}_{\mathrm{i}+1}$ are simultaneously "high" and the pre-driver serializes the 4-bit data and drives them towards the main driver. Subsequently, the main driver transmits the serialized data. The eye diagram size can be adjusted to be asymmetric by varying the number of the enabled TX cells and by controlling the bias level for the tail current of the enabled main driver. For instance, one TX cell is designed to adjust a voltage value of $37.5 \mathrm{mV}$ for this interface with a parallel termination for the TX and RX. Further, the tail current of the main driver is digitally controlled by a bias circuit with a 3-bit DAC.

The RX part consists of 4 RX cells for a QDR, a PLL identical to that in the TX part, and a mux to select the signal for measurement. The RX cell has the same architecture as the conventional receiver architecture, as shown in Figures 2 and 3. When the eye diagram size is asymmetric, the values of reference voltages are digitally controlled from $\mathrm{REF}_{\mathrm{H}}$ and $\mathrm{REF}_{\mathrm{L}}$ to $\mathrm{REF}_{\mathrm{H}}{ }^{\prime}$ and $\mathrm{REF}_{\mathrm{L}}{ }^{\prime}$, respectively.

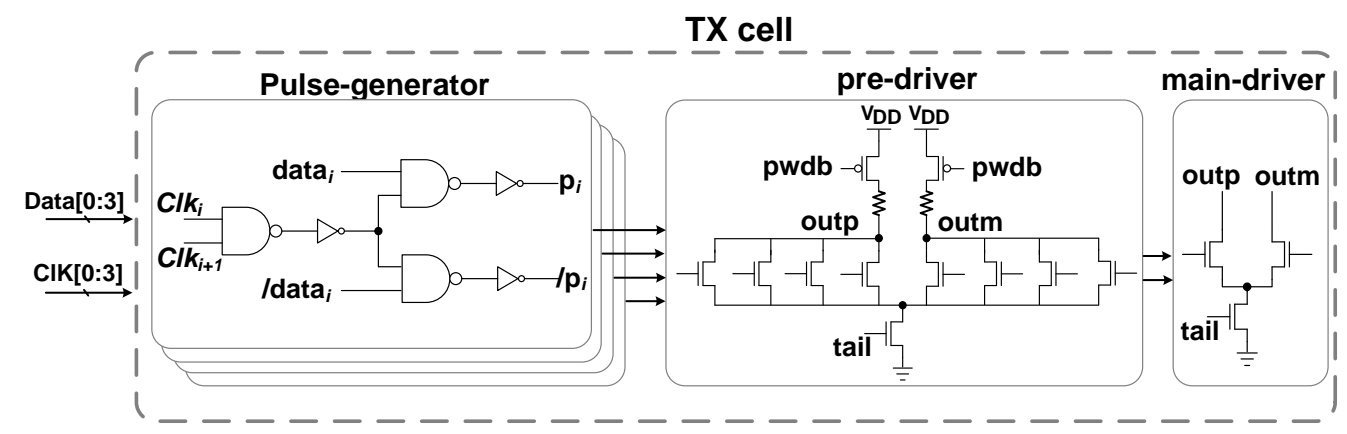

Figure 8. The schematic of TX cell 
Figure 9 shows the block diagram of the PLL for both the TX and the RX parts. It consists of the conventional charge pump PLL with a regulated voltage-controlled oscillator (VCO). The divider ratio is set at 10 .

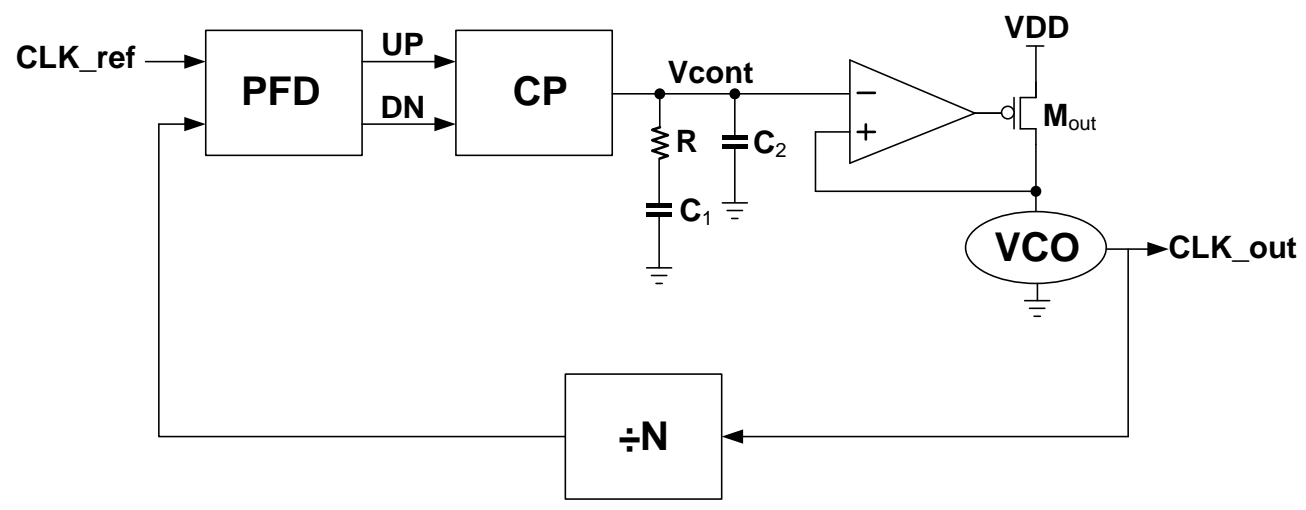

Figure 9. Block diagram of PLL

\subsection{Simulation Results}

The data rate of the proposed asymmetric 4-PAM transceiver was targeted to be 5.6$\mathrm{Gb} / \mathrm{s} / \mathrm{channel}$ with a maximum operating data rate of $6.4-\mathrm{Gb} / \mathrm{s} / \mathrm{ch}$ annel.

Figure 10 shows the simulation results of the conventional 4-PAM and the proposed asymmetric 4-PAM scheme. The simulation was achieved in the point-to-point channel with a length of $30 \mathrm{~cm}$ with a data rate of $6.4-\mathrm{Gb} / \mathrm{s} / \mathrm{channel}$, maximum operating data rate, by using HSPICE. Figure 10(a) shows the voltage margin for the eye mask of the rectangle with a time margin of about 62.5 ps. Figure 10(b) shows the time margin for the eye mask of the diamond. Table 1 summarizes the simulation results shown in Figure 10. The voltage and time margins of the asymmetric 4-PAM increased when compared with those of the conventional 4-PAM in which the reference noise exists in the RX. Generally, the magnitude of the RX reference noise is known to be about $30 \mathrm{mV}$ in a chip-to-chip interface.

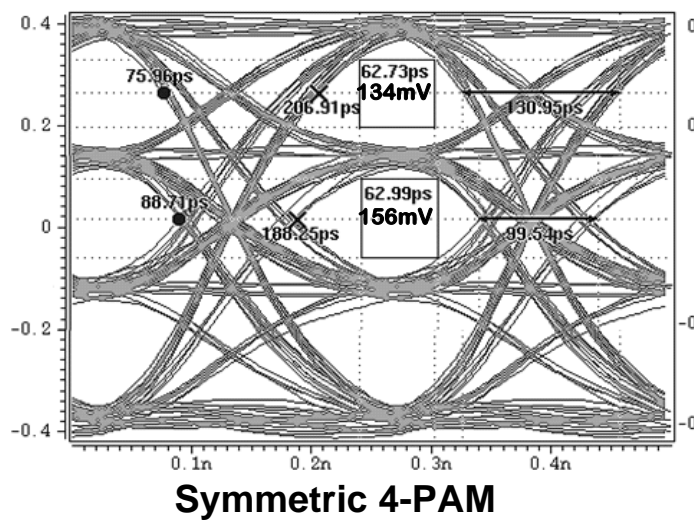

(a)

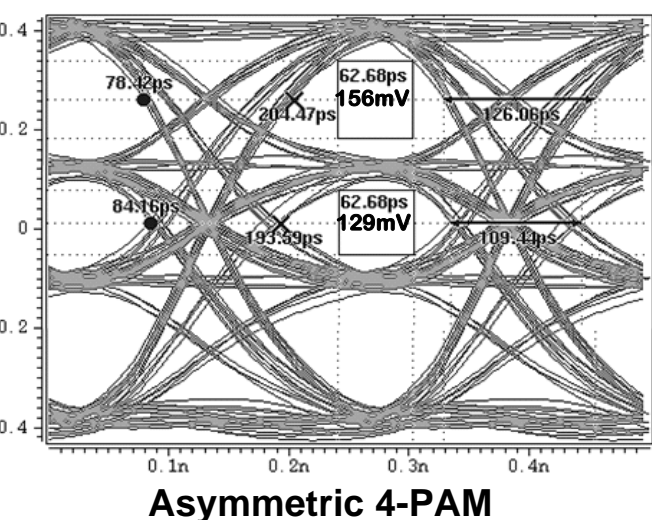

Asymmetric 4-PAM

\section{)}




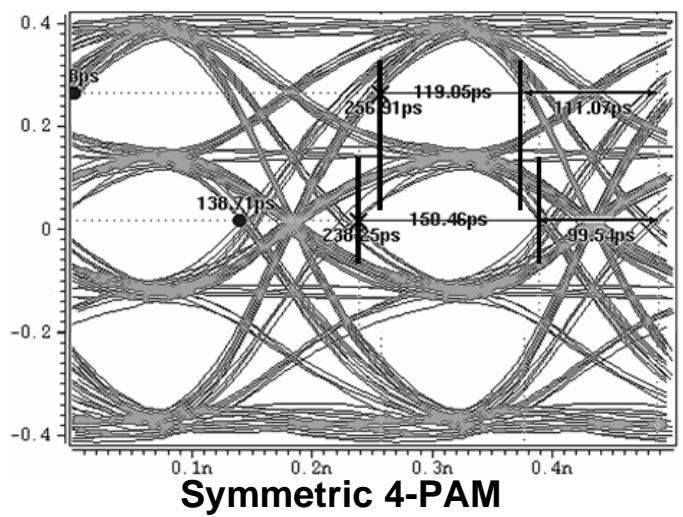

Symmetric 4-PAM

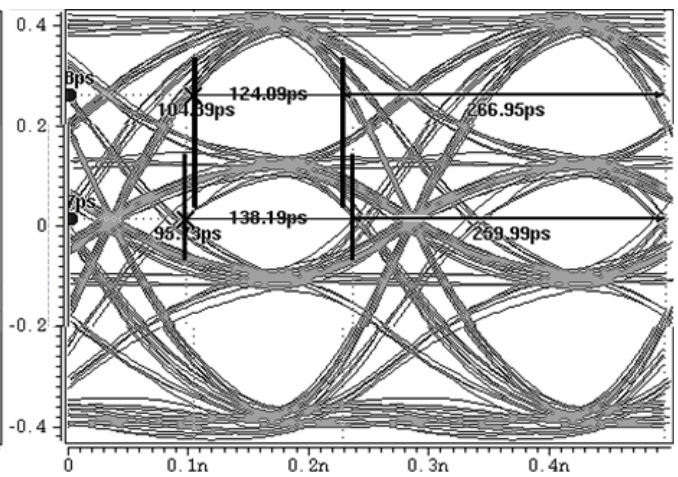

Asymmetric 4-PAM

(b)

Figure 10. Simulation results of conventional and asymmetric 4-PAM (a) voltage margin (b) time margin

Table 1. Eye margin of conventional and asymmetric 4-PAMs

\begin{tabular}{|c|c|c|c|c|c|}
\hline \multirow{2}{*}{\multicolumn{2}{|c|}{ Eye mask : Rectangle }} & \multirow{3}{*}{$\begin{array}{r}\text { eye_center } \\
156\end{array}$} & \multicolumn{3}{|c|}{ eye_upper } \\
\hline & & & \multirow{2}{*}{\begin{tabular}{|r|}
$\begin{array}{c}\text { diff_ref. noise } \\
(00 \mathrm{mV})\end{array}$ \\
134
\end{tabular}} & \multirow{2}{*}{\begin{tabular}{|c}
$\begin{array}{c}\text { diff_ref. noise } \\
(30 \mathrm{mV})\end{array}$ \\
104
\end{tabular}} & \multirow{2}{*}{$\begin{array}{l}\text { diff_ref. noise } \\
\begin{array}{l}(60 \mathrm{mV}) \\
\end{array}\end{array}$} \\
\hline \multirow{2}{*}{$\begin{array}{c}\text { voltage margin } \\
{[\mathrm{mV}]}\end{array}$} & $\begin{array}{c}\text { Symmetric } \\
\text { 4-PAM }\end{array}$ & & & & \\
\hline & \begin{tabular}{|c|} 
Asymmetric \\
4-PAM
\end{tabular} & 129 & 156 & 126 & 96 \\
\hline \multirow{2}{*}{$\begin{array}{c}\text { time margin } \\
{[\mathrm{ps}]} \\
\text { (target }: 62.5 \mathrm{ps})\end{array}$} & $\begin{array}{l}\text { Symmetric } \\
\text { 4-PAM }\end{array}$ & 62.99 & \multicolumn{3}{|c|}{62.73} \\
\hline & \begin{tabular}{|c|} 
Asymmetric \\
4-PAM
\end{tabular} & 62.68 & \multicolumn{3}{|c|}{62.68} \\
\hline \multirow{2}{*}{\multicolumn{2}{|c|}{ Eye mask : Diamond }} & \multirow[b]{2}{*}{ eye_center } & \multicolumn{3}{|c|}{ eye_upper } \\
\hline & & & \begin{tabular}{|c|} 
diff_ref. noise \\
$(00 \mathrm{mV})$
\end{tabular} & $\begin{array}{c}\text { diff_ref. noise } \\
(30 \mathrm{mV})\end{array}$ & $\begin{array}{c}\text { diff_ref. noise } \\
(60 \mathrm{mV})\end{array}$ \\
\hline \multirow{2}{*}{$\begin{array}{c}\text { voltage margin } \\
{[\mathrm{mV}]}\end{array}$} & \begin{tabular}{|c|} 
Symmetric \\
4-PAM
\end{tabular} & 214.6 & 217.4 & 187.4 & 157.4 \\
\hline & $\begin{array}{c}\text { Asymmetric } \\
\text { 4-PAM }\end{array}$ & 182.8 & 241.9 & 211.9 & 181.9 \\
\hline \multirow{2}{*}{$\begin{array}{l}\text { time margin } \\
{[\mathrm{ps}]}\end{array}$} & \begin{tabular}{|c|} 
Symmetric \\
4-PAM
\end{tabular} & 150.46 & \multicolumn{3}{|c|}{119.05} \\
\hline & \begin{tabular}{|c|} 
Asymmetric \\
4-PAM
\end{tabular} & 138.19 & \multicolumn{3}{|c|}{124.09} \\
\hline
\end{tabular}

\section{Chip Implementation and Measurement Results}

The proposed asymmetric 4-PAM transceiver was fabricated by using a $0.13-\mu \mathrm{m} 1$-poly 6 metal CMOS process with a $1.2 \mathrm{~V}$ supply. Figure 11 shows the micrograph of the TX and RX. The active area of the PLL is $500 \times 400 \mu \mathrm{m}^{2}$, and the areas of the TX and RX are $450 \times 140$ $\mu \mathrm{m}^{2}$, and $240 \times 130 \mu \mathrm{m}^{2}$, respectively. The power consumption of the 1-channel transceiver including a PLL with a $1.2 \mathrm{~V}$ supply $(\mathrm{VDDQ}=1 \mathrm{~V})$ is $6 \mathrm{~mW} / \mathrm{Gb} / \mathrm{s} / \mathrm{channel}$. 


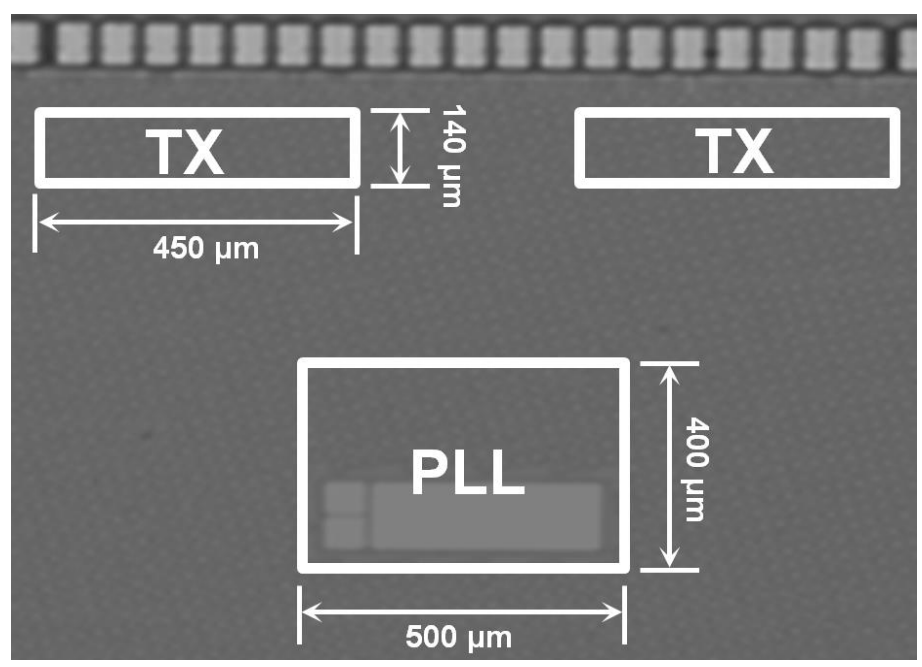

Figure 11. Chip micrograph (a) transmitter including PLL (b) receiver including PLL

The measured operating frequency range of the PLL is from $200 \mathrm{MHz}$ to $1.2 \mathrm{GHz}$. The rms and peak-to-peak jitter of the charge pump PLL are measured as $4.18 \mathrm{ps}$ and $29.8 \mathrm{ps}$, respectively, in the operating frequency of $700 \mathrm{MHz}$ for $5.6-\mathrm{Gb} / \mathrm{s} / \mathrm{channel}$ with a QDR, as shown in Figure 12.

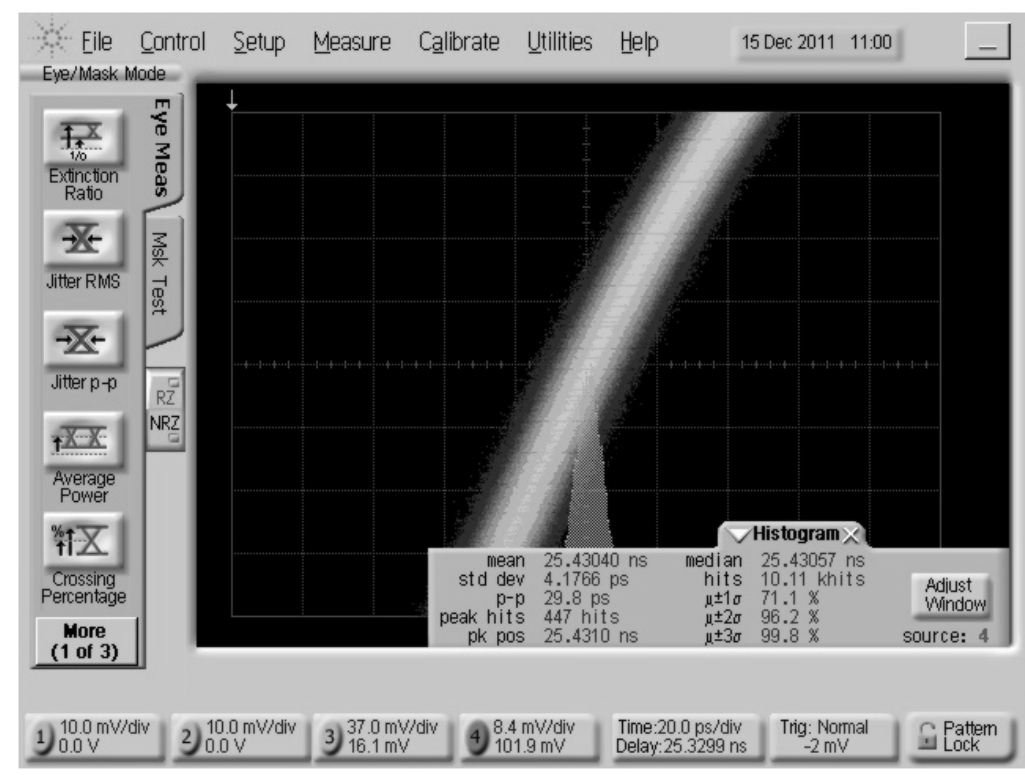

Figure 12. Measured jitter histogram of the PLL (at $700 \mathrm{MHz}$ operating frequency and $1.2 \mathrm{~V}$ supply) 


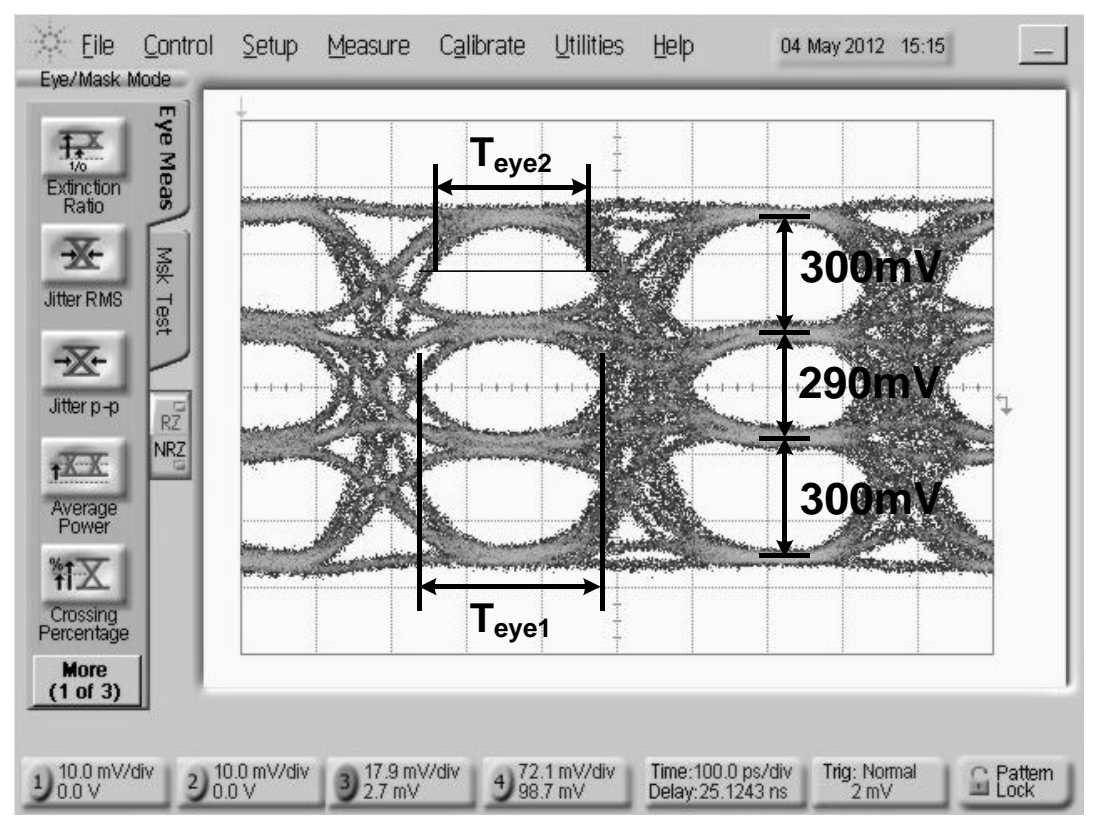

(a)

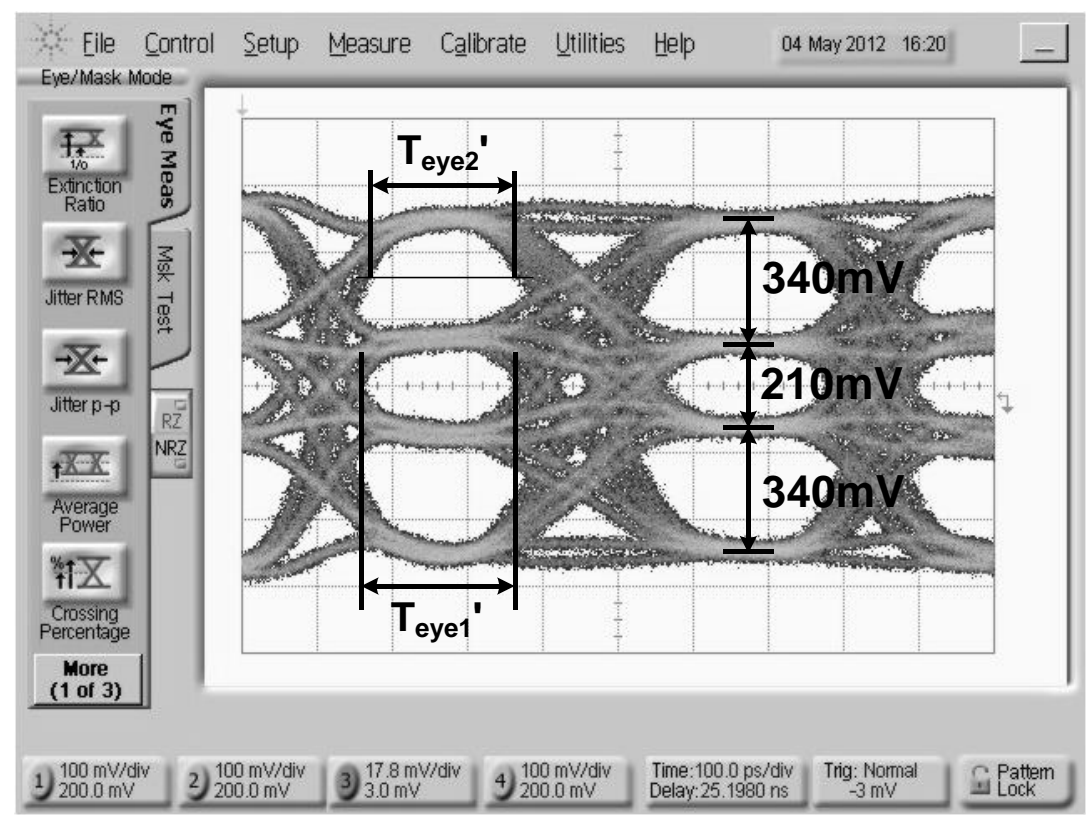

(b)

Figure 13. Measured transmit eye diagram (at $5.6 \mathrm{~Gb} / \mathrm{s})$ (a) symmetric 4-PAM (b) asymmetric 4-PAM

The eye diagrams of the symmetric and asymmetric 4-PAM with a data rate of $5.6 \mathrm{~Gb} / \mathrm{s}$ measured at the receiver section are shown in Figure 13. The measurement was achieved by using a $\mu$-stripline over $30 \mathrm{~cm}$ FR4 traces on a four-layer PCB. In the symmetric 4-PAM signaling, the magnitudes of the upper, center, and lower eyes were measured to be $300 \mathrm{mV}$, $290 \mathrm{mV}$, and $300 \mathrm{mV}$, respectively. If the noise magnitude of the single reference is $65 \mathrm{mV}$, 
the magnitude of the eye for the worst case is determined to be $170 \mathrm{mV}$ since the worst case noise magnitude of the differential reference is $130 \mathrm{mV}$. However, in the proposed asymmetric 4-PAM signaling, the magnitudes of the upper, center, and lower eyes were measured to be $340 \mathrm{mV}, 210 \mathrm{mV}$, and $340 \mathrm{mV}$, as shown in Figure $13(\mathrm{~b})$. In this case, the magnitude of eye for the worst case is determined to be $210 \mathrm{mV}$ without reduction in time margin when the noise magnitude of the single reference is $65 \mathrm{mV}$. As a result, the voltage margin is increased by $2 \alpha$, about $40 \mathrm{mV}$. Furthermore, the time margin did not decrease due to use of the proposed asymmetric 4-PAM for the increase of the voltage margin, as shown in Figure 13. The measured time margins of the conventional and proposed asymmetric 4-PAM were determined to be the time margin of the upper and lower eyes $\left(\mathrm{T}_{\text {eye2 }}, \mathrm{T}_{\text {eye2 }}{ }^{\prime}\right)$. These had almost equal values by 200 and 193 ps.

\section{Conclusion}

A 5.6-Gb/s/channel asymmetric 4-PAM transceiver was designed for a display interface with ultra-high definition, and it was fabricated by using a $0.13-\mu \mathrm{m}$ CMOS process with a 1.2 $\mathrm{V}$ supply. The simulation results show that the proposed asymmetric 4-PAM reduced the effects of reference noise in a receiver by $33 \%$ and increased the time margin when compared with that of a conventional 4-PAM. The rms and peak-to-peak jitter of the charge pump PLL were measured as $4.18 \mathrm{ps}$ and $29.8 \mathrm{ps}$, respectively, in operating frequency of $700 \mathrm{MHz}$. The measurement results show that the magnitude of the eye for the worst case is determined to be $210 \mathrm{mV}$ when the magnitudes of the upper, center, and lower eyes are $340 \mathrm{mV}, 210 \mathrm{mV}$, and $340 \mathrm{mV}$, and the noise magnitude of the single reference is $65 \mathrm{mV}$. As a result, the proposed asymmetric 4-PAM signaling increases the voltage margin by $23.5 \%$ without reduction in the time margin when compared with the corresponding values for conventional 4-PAM signaling when the noise magnitude of the single reference is $65 \mathrm{mV}$. The power consumption of the proposed transceiver was $6 \mathrm{~mW} / \mathrm{Gb} / \mathrm{s} / \mathrm{channel}$.

\section{Acknowledgements}

This paper was supported by Research Fund, Kumoh National Institute of Technology.

\section{References}

[1] K.-Y. K. Chang, J. Wei, C. Huang, S. Li, K. Donnelly, M. Horowitz, Li Yingxuan and S. Sidiropoulos, "A 0.4-4Gb/s CMOS quad transceiver cell using on-chip regulated dual-loop PLLs”, IEEE J. Solid-State Circuits, vol. 38, no. 5, (2003) May, pp. 747-754.

[2] N. Nhat, Y. Frans, B. Leibowitz, S. Li, R. Navid, M. Aleksic, F. Lee, F. Fredy Quan, J. Zerbe, R. Perego and F. Assaderaghi, "A 16-Gb/s differential I/O cell with 380fs RJ in an emulated 40nm DRAM process", IEEE VLSI Circuit Symp., (2008) June, pp. 128-129.

[3] K. -h. Kim, H. -J. Chung, W. -S. Kim, M. Park, Y. -C. Jang, J. -Y. Kim, H. -W. Park, U. Kang, P. W. Coteus, J. S. Choi and C. Kim, "An 8Gb/s/pin 9.6ns Row-Cycle 288Mb Deca-Data Rate SDRAM with an I/O ErrorDetection Scheme”, IEEE J. Solid-State Circuits, vol. 42, no. 1, (2007) January, pp. 193-200.

[4] J. Zerbe, C. Werner, V. Stojanovic, F. Chen, J. Wei, G. Tsang, D. Kim, W. Stonecypher, A. Ho, T. Thrush, R. Kollipara, G.-J. Yeh, M. Horowitz and K. Donnelly, "Equalization and Clock Recovery for a 2.5-10Gb/s 2PAM/4-PAM Backplane Transceiver Cell", IEEE J. Solid-State Circuits, vol. 38, no. 12, (2003) December, pp. 2121-2130.

[5] J. F. Buckwalter, M. Meghelli, D. J. Friedman and A. Hajimiri, "Phase and amplitude pre-emphasis techniques for low-power serial links," IEEE J. Solid-State Circuits, vol. 41, no. 6, (2006) June, pp. 13911399. 


\section{Authors}

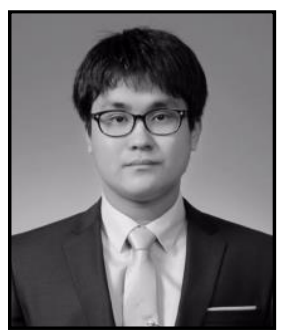

\section{Kwang-Hun Lee}

He was born in Gumi, Korea, in 1985. He received the B.S. and M.S. degrees in electronic engineering from Kumoh National Institute of Technology, Gumi, Korea, in 2011 and 2013, respectively. He joined SK Hynix Inc. Gyeonggi-do, Korea, in 2013. His current research interests are in the design of PLL, DLL, and High speed interface.

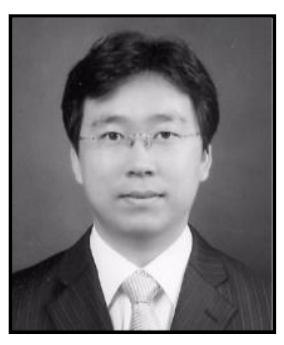

\section{Young-Chan Jang}

He was born in Daegu, Korea, in 1976. He received the B.S. degree in electronic engineering from Gyeongbuk National University, Daegu, Korea, in 1999 and the M.S. and Ph.D. degrees in electronic engineering from Pohang University of Science and Technology (POSTECH), Pohang, Korea, in 2001 and 2005, respectively. From 2005 to 2009, he was a Senior Engineer in the Memory Division, Samsung Electronics, Hwasung, Korea, working on high-speed interface circuit design and next-generation DRAM. In 2009, he joined the Faculty of School of Electronic Engineering, Kumoh National Institute of Technology, Gumi, Gyeongbuk, Korea, where he is currently an Associate Professor. His current research area is high-performance mixed-mode circuit design for VLSI systems such as high-performance signaling, clock generation, and analog-to-digital conversion. 
International Journal of Control and Automation Vol.6, No.6 (2013) 